\title{
REGION OF INTEREST DETECTION BASED ON HISTOGRAM SEGMENTATION FOR SATELLITE IMAGE
}

\author{
Warinthorn Kiadtikornthaweeyot ${ }^{\mathrm{a}}$, Adrian R.L. Tatnall ${ }^{\mathrm{b}}$ \\ ${ }^{a}$ Geo-Informatics and Space Technology Development Agency, 20 The Government Complex, Building 6th-7th Floor, \\ Chaeng Wattana Road, Lak Si, Bangkok, 10210 - warinthorn@ gistda.or.th \\ ${ }^{\mathrm{b}}$ University of Southampton, University Road, Highfield, Southampton SO17 1BJ, UK - art@ soton.ac.uk
}

Commission WG VII/4

KEY WORDS: Region of interest, Image segmentation, Histogram segmentation, Morphological, Satellite image

\begin{abstract}
:
High resolution satellite imaging is considered as the outstanding applicant to extract the Earth's surface information. Extraction of a feature of an image is very difficult due to having to find the appropriate image segmentation techniques and combine different methods to detect the Region of Interest (ROI) most effectively. This paper proposes techniques to classify objects in the satellite image by using image processing methods on high-resolution satellite images. The systems to identify the ROI focus on forests, urban and agriculture areas. The proposed system is based on histograms of the image to classify objects using thresholding. The thresholding is performed by considering the behaviour of the histogram mapping to a particular region in the satellite image. The proposed model is based on histogram segmentation and morphology techniques. There are five main steps supporting each other; Histogram classification, Histogram segmentation, Morphological dilation, Morphological fill image area and holes and ROI management. The methods to detect the ROI of the satellite images based on histogram classification have been studied, implemented and tested. The algorithm is be able to detect the area of forests, urban and agriculture separately. The image segmentation methods can detect the ROI and reduce the size of the original image by discarding the unnecessary parts.
\end{abstract}

\section{INTRODUCTION}

The satellite image is the most important tool to extract the Earth's surface information. There are many human activities of interest such as residential developments or suburban areas or the percentage of the forest in the selected area; these can be studied from the satellite images. Remote sensing imaging has been applied in many application fields such as land-use and land-cover in the last two decades. Moreover satellite imaging is able to compute estimates of agricultural areas due to being able to cover a wide region (Soontranon et al, 2015).

More than half of the world's population live in Asia, and this is also the major area to produce rice. Monitoring, mapping and forecasting paddy rice agriculture can lead to efficiency in managing food and water production (Xial et al., 2006), (Y.H. Tseng et al, 1998). The main agriculture product of Thailand is rice. Geo-Informatics and Space Technology Development Agency (GISTDA) has launched Agriculture Monitoring System (AMS-Thailand) since 2012 (Soontranon et al. 2012). The rice field area must be classified from other plants, therefore in this paper the technique to classify the object in the optical satellite had been studied and tested.

A real scene observed from a satellite image contains a variety of features, textures and shadows and it can therefore be very complex to detect the region of interest (ROI). The interesting part of a satellite image depends on the application field for Earth observation. Therefore image segmentation has been developed for extracting different features or textures inside an image. This can be performed a number of different ways using the image properties. This paper proposes the use of histogram segmentation techniques to classify the object in the optical satellite image. The final target of this research is to detect the rice fields in Thailand and also increase the accuracy of the automation.

The rest of the paper is organized as follows: section 2 describes the ROI automatic detection based on histogram segmentation. Section 3 presents the proposed system and the process of the model step by step. Section 4 demonstrates the experiment and results. The final section gives a conclusion and identifies future work.

\section{ROI AUTOMATIC DETECTION BASED ON HISTOGRAM SEGMENTATION}

\subsection{Automatic detection of Region of Interest}

Image segmentation is a highly important tool in image analysis. To identify the object in the image the ROI detection has been applied in many areas, for example, medical imaging, security surveillance, database and remote sensing imaging (Rajkumar, 2001) and (Fei, 2007). Classification of the ROI of an image can be performed using a manual or automatic process. There have been many studies to develop automatic ROI detection by using image segmentation techniques (Guamieri, 2002; Oscal, 2007 ;Guang, 2009 and Shinji, 2005). The level of image segmentation depends on the final solution that will be applied in the application. There are many different techniques had been proposed to detect ROI. For example, The Hough transform was originally proposed by Paul hough (Ballard, 1981), Region of interest image classification using a Zernike moment signature was represented (Wee, 2007).

Extraction of the required ROI from within an image is very difficult using only one image segmentation technique due to 
satellite image complexity. The effective solution is to use a combination of different image processing techniques to identify the region of interest. There are many image segmentation methods, which are often based on basic properties of intensity values, discontinuity and similarity (Rafael, 2008; Salem, 2010 ; Cheng, 2001 and Spirkorska, 1993). This paper identifies the combination of the suitable image segmentation techniques that can be used to detect the ROI of the satellite image and enable classification as forests, urban and agriculture areas.

\subsection{Histogram segmentation}

The ROI of the satellite image depends on the Earth observation application fields. Some applications are concerned with identifying one object of interest.

There are a number of different techniques to identify the clusters corresponding to ROI. Supervised classification techniques which rely on labelled reference samples have been applied to classify the satellite image (Bruzzone and Persello 2009). In addition active learning, neural networks and support vector machines have all successfully been used to classify imagery (Tuia, Volpi et al. 2011; Xiuming and Jing 2011; Crawford, Tuia et al. 2013).

In this paper, the histogram segmentation method is based on threshold classification. The objects in the image are separated into the different groups according to the intensity of the pixel. This research classifies the object by labelling three groups; urban, forest and agriculture using referenced samples. The thresholding is considered by each peak of the histogram and mapping to the region of object. Each thresholding is separated manually based on the information of the image.

\section{THE PROPOSED SYSTEM ALGORITHM}

The pixel values in an image can be represented by histograms. The concept of histogram segmentation is classifying an image using thresholds. The thresholding is performed by considering each peak of the histogram and mapping to a particular region by the concept of different intensities matching to different regions (Kurugollu, 2001). The flow chart in figure 1 illustrates the proposed ROI automatic detection based on histogram image segmentation algorithm. There are five sub-processes in the proposed system;

- Histogram classification

- Histogram segmentation

- Black and white image conversion

- Application of morphological techniques

- ROI code management

The process starts with receiving the satellite image. In this paper the optical satellite image is used (such as image in Figure 2). Figure 2 shows the input image, which is located in Burghause, Germany. It was taken by RapidEye with 5 meter resolution of multi-spectra band image (Romeijn, 2011). The image are RGB image to test. Firstly the histogram and grouping of pixels in the object by considering each peak of the histogram is performed. The thresholding is then applied to the image to convert it to a binary image identifying pixels of interest and not of interest.

The next step is to apply dilation morphology and fill the holes of the image to get the ROI mask. The final step is to apply the ROI mask to the original image to extract the ROI image as the output image.

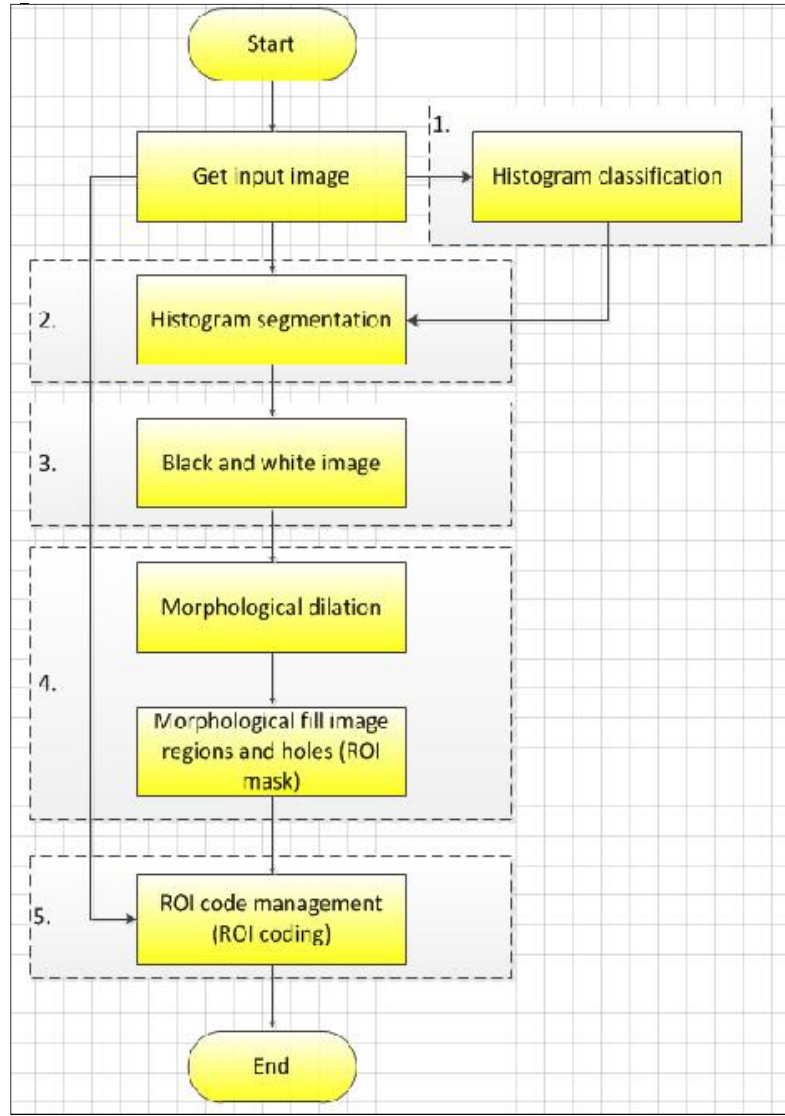

Figure 1. The propose algorithm for ROI automatic detection base on histogram segmentation.

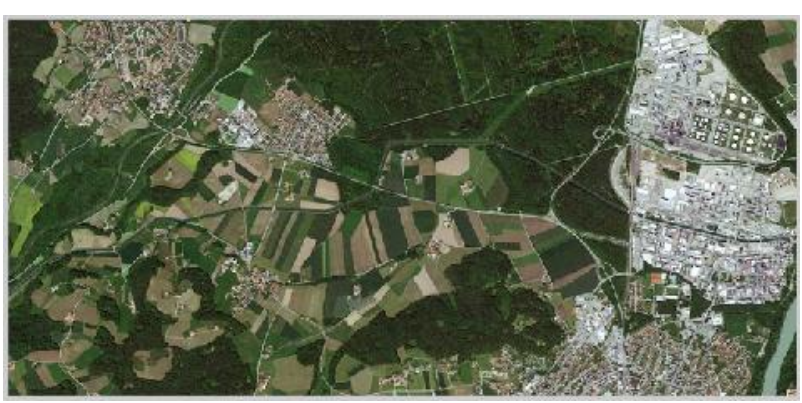

Figure 2. Input image for the experiment

The process of histogram segmentation has been described for each sub-process as more detailed below;

\subsection{Histogram classification}

The input image has been converted from RGB image in to grayscale image. This process converts the brightness of each pixel to a grey level, which usually consists of 256 levels. Each of the RGB channels could be used to generate a grey level image individually but using a combination of the channels can prove useful. As an example the constants used for each channel shown in Equation (1) results in a better approximation of the human visualizer's sensitivity to each of those colours (Kanan and Cottrell 2012). $l_{g}$ is the grey scale image, and $l_{r}, l_{g}$ and $l_{t}$ represent the image pixel value in red, green and blue channels respectively. 


$$
\mathrm{I}_{\text {grey }}=\left(\mathrm{I}_{r} \times 0.30\right)+\left(\mathrm{I}_{g} \times 0.59\right)+\left(\mathrm{I}_{b} \times 0.11\right)
$$

Objects in the image are separated into the different groups according to the intensity of the pixel. There are a number of different techniques to identify the clusters corresponding to ROI. The process to classify the objects in the image to different groups has been developed as detailed in the following:

For the initial design the threshold is calculated by the random selection of pixels for each object and the identification of the appropriate group by human intervention. This sampling information is analysed for statistical features such as the maximum, minimum and average values. The threshold is set based on these statistical features of each group for the example pixels, and the test data recorded is shown in table 1.

\begin{tabular}{|c|c|c|c|}
\hline \multirow{2}{*}{$\begin{array}{c}\text { Data } \\
\text { Record 500 } \\
\text { samples }\end{array}$} & \multicolumn{3}{|c|}{ Pixel value } \\
\cline { 2 - 4 } & Forest & Agriculture & Urban \\
\hline Average & 58.75 & 119.65 & 185.40 \\
\hline Max & 75 & 155 & 234 \\
\hline Min & 25 & 91 & 163 \\
\hline
\end{tabular}

Table 1. The data record of the pixels value for each object.

Secondly, this segmentation process is based on the image grey level histogram as shown in figure 3. The statistical values from the first process are applied to the histogram image to compare the intensity range of each object. After obtaining the minimum and maximum intensity of each object, these thresholds (histogram thresholding set) are applied to the histogram segmentation (next process). For example the values of pixels of the forest are between 25 to 75 and from the test the average pixel value of the forest is 58.75. If pixels are classified as agriculture they have values between 91 to 155 and the average pixel value is 119.65 . Therefore, some pixel values are not classified in to any group. Those pixels that are not classified as any group are identified by the white colour in the histogram segmented image. The assumption is that the objects are characterised uniquely by their brightness. In practice this will not be the case but it provides a starting point for the analysis.

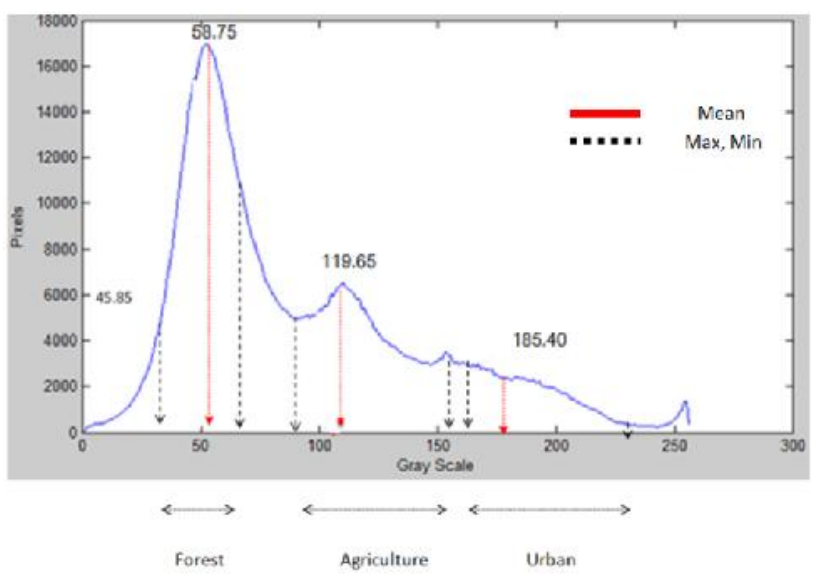

Figure 3. Grayscale histogram image and thresholding set

The process of thresholding classification classifies the object with manual thresholding, i.e. the thresholding is done by manual intervention. However for the final design, the histogram classification model needs to be developed to be automatic by clustering data algorithm.

\subsection{Histogram segmentation:}

Every pixel of the original image is compared with the histogram thresholds set and allocated to a group if possible. The pixels belonging to each group are represented by different colours. Figure 4 shows the example of the output image after processing by histogram segmentation for forest detection. The pixels are classified into three categories; forest, agriculture and urban, which are represented by green, blue and red respectively.

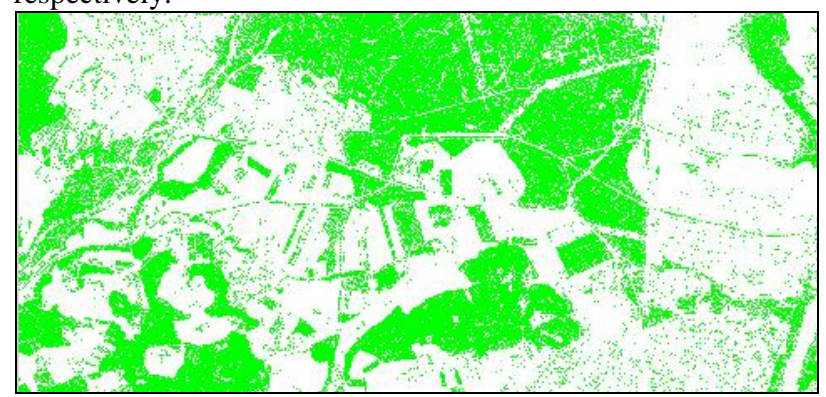

Figure 4. Forest classified by Histogram segmentation.

Figure 5 shows the histogram segmented image. There are 3 classified areas; forest, agriculture and urban. The pixels which are not classified as any object are shown in white.

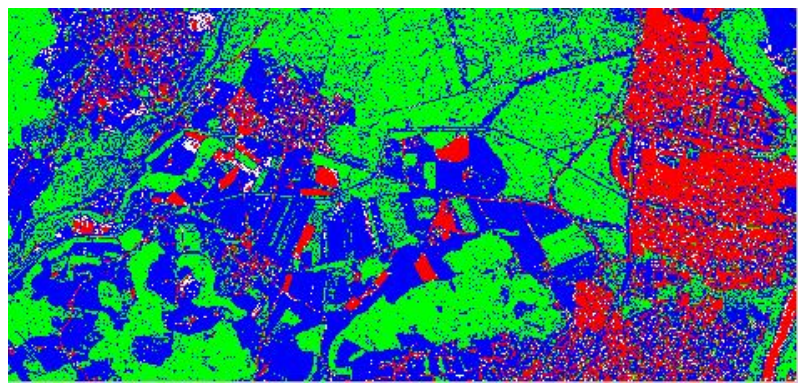

Figure 5 . The histogram segmented image by the proposed system

\subsection{Black and white image conversion:}

This process converts the histogram segmented image to a binary image. The image has its pixels replaced by either a black or white colour depending on whether the pixel is in a classified category in a region of interest. The input image pixels are replaced with the value ' 1 ' (white) if the input pixels are in ROI areas, i.e. forest, urban and agriculture, whereas other pixels are replaced with the value ' 0 ' (black) if they are categorised as the not interested area. Figure 6 shows the conversion of the histogram segmented image, to a black and white image. In this example using the manual histogram classification, there are some pixels, which are not classified to any group and produced some error in the classification. 


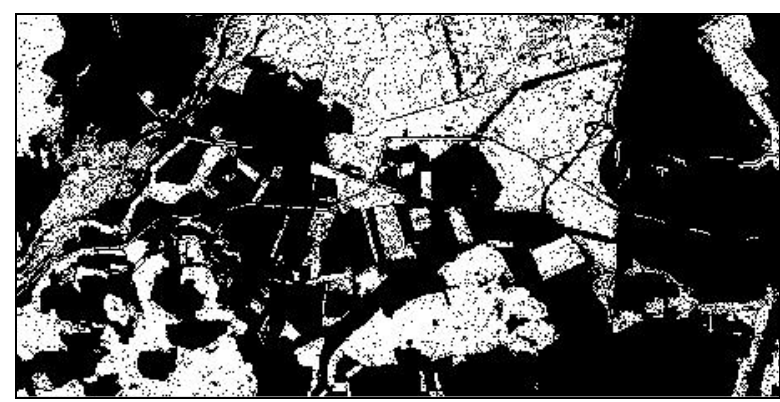

Figure 6. Example of black and white image conversion.

\subsection{Mathematical morphology:}

This process consists of two morphology techniques; 'dilation' and 'fill the regions and holes'.

- The dilation morphology technique: After the histogram segmentation, a mathematical morphology technique is used to connect areas which are separated by smaller spaces than that of the structuring element used. The dilation which is implemented using this method gradually enlarges the boundaries of regions of objects. The dilation operator has two data inputs. The first one is the image and the second one is the structure element which is defined by size and shape (Ves, Benavent et al. 2006; Gonzalez and Woods 2007). A considerable amount of research has been performed on the morphology of the structuring elements (Hedberg, Dokladal et al. 2009; Wang 2009). The selection of a structuring element size and shape depends on the geometric shapes that need to be extracted from the input image. For instance, for extracting shapes from geographic aerial images of a city, a square or rectangular element will provide a good result. On the other hand, line structure elements are good at detecting roads in an image

- $\quad$ Fill the holes technique: The image from the previous step is then processed by the region and hole filling technique. This technique fills image regions and holes and enables a boundary line to be identified (Xiao-hong, Zhu et al. 2009). The process works by detection of an area of dark pixels surrounded by white pixels. The ROI mask is generated in this step. The technique is based on dilation, complementation and intersection for filling holes (Gonzalez and Woods 2007; Al-amri, Kalyankar et al. 2010). Figure 7 demonstrates the result after image dilation and fill holes has been performed.

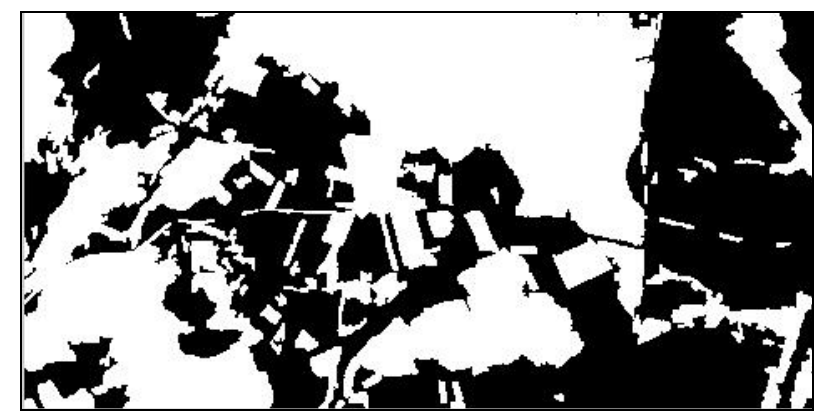

Figure 7. Example of dilated and fill holes image.

\subsection{ROI code management:}

This process applies the ROI mask to the original image by comparison of the two images. The parts of the original image, which are identified by the ROI mask, will be transferred to the final image. On the other hand, the part of original image which is marked by the non-ROI mask will be replaced by ' 0 ' in the final image (i.e. those areas not of interest). Figure 8 shows the output ROI automatic detection image; Forest are the interesting area and other areas are non-ROI.

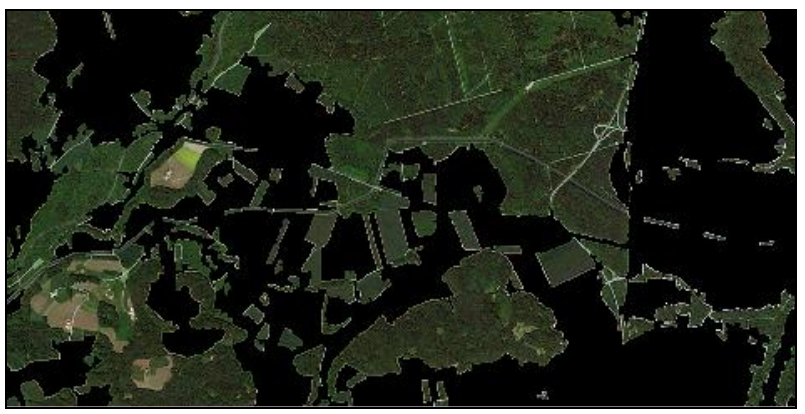

Figure 8. ROI detection (Forest) by the proposed technique.

\section{EXPERIMENT AND RESULT}

This section presents the result of each the process to detect the ROI of the image, which consists of one specific feature using the proposed histogram segmentation technique. There are three tests that have been performed, to detect forest, agriculture and urban. Figure 3 shows the histogram of the image grayscale with the sampling set of each object with the minimum, maximum and average value of the sampling set. Table 2 shows the result of each process to detect the forest in the image. First the process starts with converting the image from the RGB image to a grayscale image. The second process applies the histogram threshold of the forest to the grayscale image and the pixels that are in the range of the forest are labelled green as show in the Table 2 'Histogram segmented image'. The next process is morphological dilation to connect close small areas in the same area of interest together. The application of the morphological fill of regions and holes, gives the ROI mask at the end of this process. The final step is to apply the ROI mask to the original image to produce the ROI image.

Table 3 and 4 show the images that result from each step required to detect the urban and agriculture in the image respectively. 


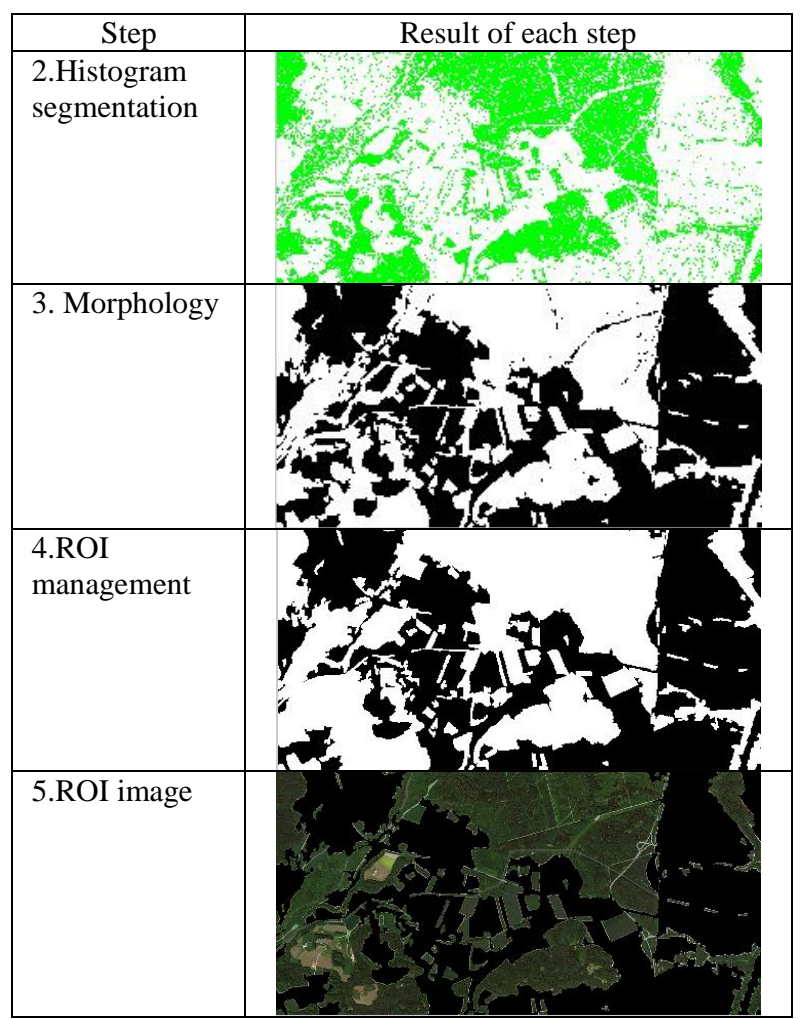

Table 2. The result of the proposed system of each process to detect only forest in the sample

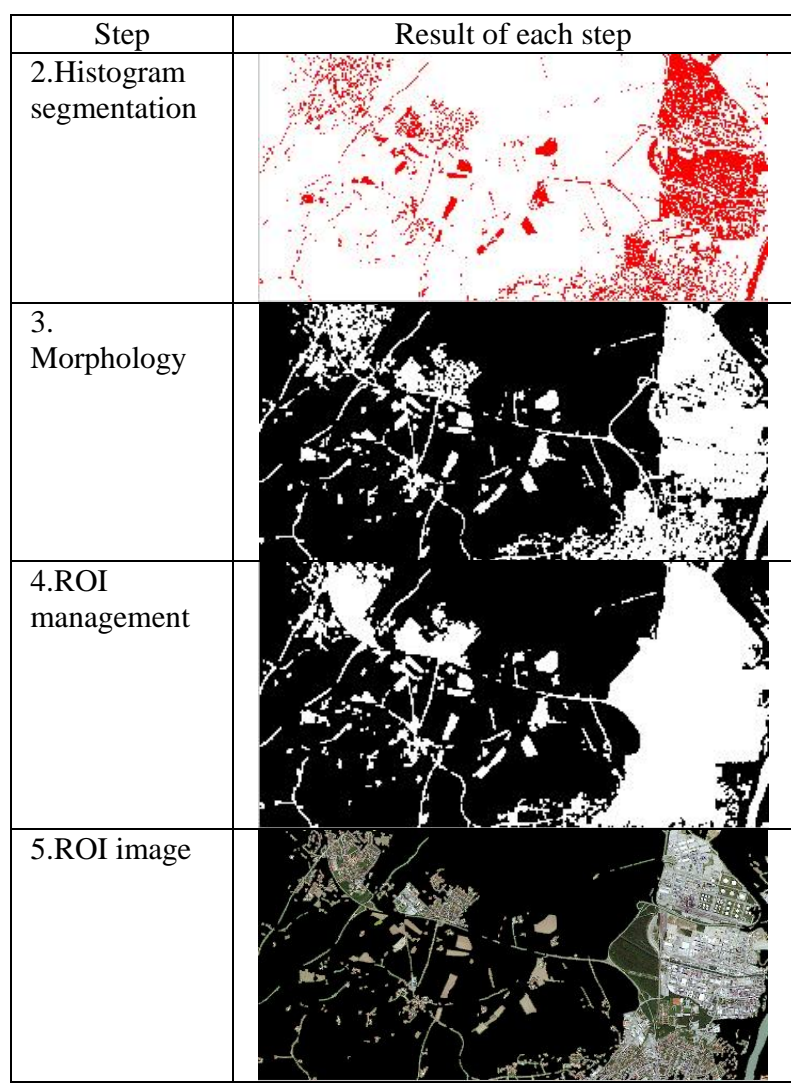

Table 3. The result of the proposed system of each process to detect only urban in the sample image

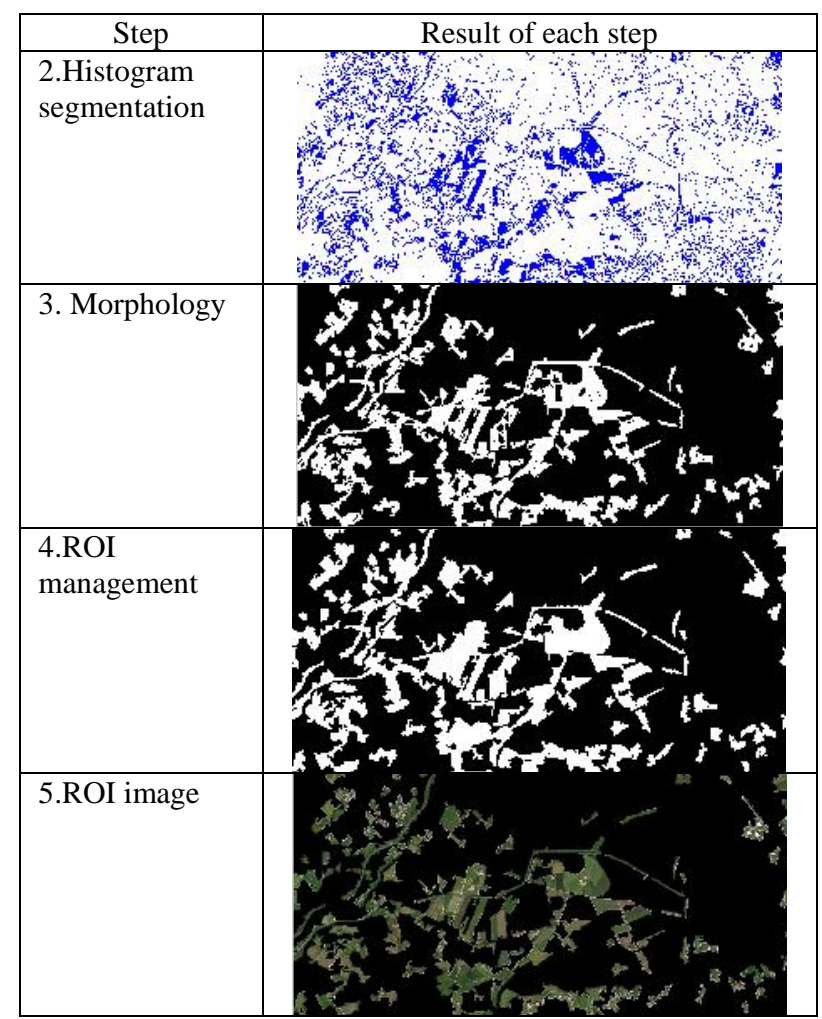

Table 4 . The result of the proposed system of each process to detect only agriculture in the sample image

Table 5 shows the portion of each object size of the image, which are detected using the proposed system on a specific object of interest. The ROI images for forest, agriculture and urban have been found in the portions of $48.47 \%, 26.89 \%$ and $40.16 \%$ of the original image respectively. The test result shows that the ROI automatic detection based on histogram segmentation is possible to detect the specific object in the image as the ROI object. Moreover if some applications only requires the ROI area of the image, then this is inherently a smaller size compared with the original image, and is therefore now more convenient to store or transfer.

\begin{tabular}{|l|c|c|c|}
\hline \multirow{2}{*}{$\begin{array}{c}\text { ROI detection } \\
\text { object }\end{array}$} & \multicolumn{3}{|c|}{$\begin{array}{c}\text { ROI automatic detection based on } \\
\text { histogram segmentation for specific } \\
\text { object }\end{array}$} \\
\cline { 2 - 4 } & $\begin{array}{c}\text { Input size : } \\
\text { A }\end{array}$ & $\begin{array}{c}\text { ROI } \\
\text { image size }\end{array}$ & $\begin{array}{c}\text { Portion of } \\
\text { each object }\end{array}$ \\
\hline 1. Forest & $2,886 \mathrm{~KB}$ & $1,399 \mathrm{~KB}$ & $48.47 \%$ \\
\hline 2.Agriculture & $2,886 \mathrm{~KB}$ & $776 \mathrm{~KB}$ & $26.89 \%$ \\
\hline 3. Urban & $2,886 \mathrm{~KB}$ & $1,159 \mathrm{~KB}$ & $40.16 \%$ \\
\hline
\end{tabular}

Table 5. The result of the proposed system of each process to detect only agriculture in the sample image

The correctness of the proposed system has been examined by comparing the number of pixels between manual and the proposed automatic detection, which are detected as ROI image. The ROI image of both methods are compared by counting the number of different pixels. Then using the number finds the percentage of the same pixels of ROIs image from both detections. Figure 9 shown the manual detection, which is performed by drawing the outline of the ROI by eye. Moreover, Figure 10 shown the ROI automatic detection of urban of the 
test image. For this test, the ROI images of urban have been calculate the number of the same pixels between both detection methods. The result of the test shows in Table 6. The example satellite image has been tested for three times. The percentage of the same pixels that detected as the ROI between the proposed ROI automatic and manual detection is approximately 88.87 percentages.

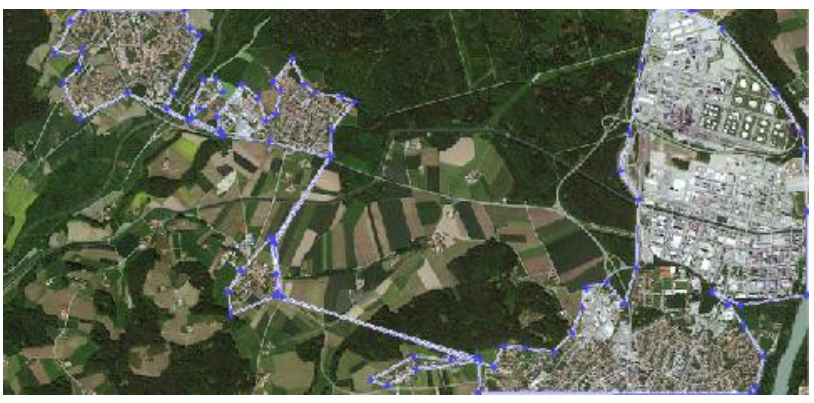

Figure 9. ROI of urban manual outline drawing.

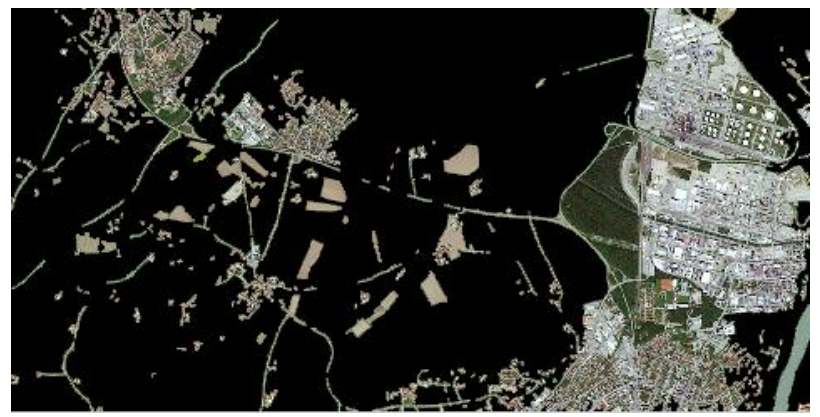

Figure 10. ROI automatic detection by the proposed system of urban of the test image.

\begin{tabular}{|c|c|c|c|}
\hline $\begin{array}{c}\text { Test } \\
\text { number }\end{array}$ & $\begin{array}{c}\text { Different } \\
\text { pixels } \\
\text { number }\end{array}$ & $\begin{array}{c}\text { Percentage of the } \\
\text { same pixels in ROI } \\
\text { of both methods }\end{array}$ & Average \\
\cline { 1 - 3 } Test 1 & 123,268 & $88.72 \%$ & \multirow{2}{*}{$88.87 \%$} \\
\cline { 1 - 3 } Test 2 & 115,994 & $89.38 \%$ & \\
\hline Test 3 & 125,576 & $88.51 \%$ & \\
\hline
\end{tabular}

Table 6. The comparison of result between ROI automatic detection and ROI manual detection

\section{CONCLUDSION AND FUTURE WORK}

This paper proposes techniques to classify the object in the satellite image by using ROI automatic detection technique based on histogram segmentation.

The ROI automatic detection is a method to define the area of an image that contains information of interest. The proposed segmentation to detect the ROI of the image has been studied, implemented and tested. The histogram method is based on thresholding segmentation and morphology dilation fill gap and hole. It is able to classify the ROI area. The histogram classification is still manually applied to manage the group of pixels. The proposed system has been verify the accuracy by compare the manual ROI detection with the automatic detection. Therefore future work is to implement automatic histogram classification from the information of the histogram of the image and also how to identify smaller and more specific ROI in an image, for example a rice field or specific patch of land.

\section{REFERENCES}

Al-amri, S. S., N. V. Kalyankar, and Khamitkar S.D., 2010 "Image Segmentation by Using Threshold Techniques." Journal of Computing. vol2 Issue 5

Ballard, D. (1981). Generalizing the hough transform to detect arbitrary shapes. Pattern Recognition, 13(2), pp111-122

Bruzzone, L. and C. Persello., 2009. Active learning for classification of remote sensing images. Geoscience and Remote Sensing Symposium,2009 IEEE International,IGARSS 2009.

Cheng H.D., Jiang X.H., Sun Y., and Jingli W., 2001. Colour image segmentation : advances and prospects. ELSEVIER, Pattern Recognition, Volume 34, Issue 12, , pp. 2259-2281

Crawford, M. M., D. Tuia, et al., 2013. "Active Learning: Any Value for Classification of Remotely Sensed Data?" Proceedings of the IEEE_101(3): 593-608.

Fei, W., W. Jishang, et al., 2007. Automatic ROI Selection for JPEG2000 Compression of Remote Sensing Images. Semantic Computing, 2007. ICSC 2007. International Conference.

Guang, H., X. Ming, et al., 2009. Detecting Automatically and Compression Algorithm for Infrared Image Based on Region of Interest. Computer Science-Technology and Applications, 2009. IFCSTA '09. International Forum on.

Guarnieri, A. and A. Vettore., 2002. Automated Techniques for satellite image segmentation. ISPRS Commission IV, Symposium 2002 on Geospatial Theory, Processing and Applications Ottawa, Canada, ISPRS Archives.

Gonzalez, R. and R. Woods., 2007. Digital Image Processing Prentice Hall.

Hedberg, H., P. Dokladal, et al., 2009. "Binary Morphology With Spatially Variant Structuring Elements: Algorithm and Architecture." Image Processing, IEEE Transactions on18(3): $562-572$.

Kanan, C. and G. W. Cottrell (2012). "Color-to-Grayscale: Does the Method Matter in Image Recognition?" PLoS ONE7(1): e29740.

Kurugollu, F., B. Sankur, et al., 2001. "Color image segmentation using histogram multithresholding and fusion." Image and Vision Computing19(13): 915-928.

Oscal, T.C., and Chih-Chang C., 2007. AutomaticallyDetermined Region of Interest in JPEG 2000. IEEE Transactions on multimedia, VOL. 9, No.7.

Rafael, C. G., and Richard, E. W., 2008. Digital Image Processing, Third Edition. Pearson Prentice Hall, Upper Saddle River, NJ 07458, US. 
Rajkumar, T. M. P. and M. V. Latte., 2011. "ROI Based Encoding of Medical Images: An Effective Scheme Using Lifting Wavelets and SPIHT for Telemedicine." International Journal of Computer Theory and Engineering3(3): 9.

Romeijn Leopold., 2011. satellite image corporation Retrieved 9 October 2015,from:

http://news.J.satimagingcorp.com/2011/06/satellite_imaging_co rporation_sic_signs_agreement_with_rapideye_ag_for_distribut ion_of_rapideye_satellite_image_data.html

Salem Saleh, A., Kalyankar, N.V., Khamitkar S.D., 2010. Image Segmentation by Using Threshold Techniques. Computer Vision and Pattern Recognition Journal of Computing, Volume 2 , Issue 5 .

Shinji, F., Toshihiko, T., and Masahiko, N., 2005. Switching Wavelet Transform for ROI Image Coding. Journal IEICE Transactions on Fundamentals of Electronics, Communications and Computer Sciences archive, Vol E88-A Issue 7, Oxford University Press Oxford, UK.

Soontranon, N., Srestasathiern, P., and Rakwatin, P., 2015b. Rice crop calendar based on phenology analysis from timeseries images. In Electrical Engineering/Electronics, Computer, Telecommunications and Information Technology (ECTICON), 2015 12th International Conference on, pages 1-5. IEEE.

Spirkovska, L.,1993. A summary of image segmentation techniques. Moffett Field, Calif. : [Springfield, Va. : National Aeronautics and Space Administration, Ames Research Center; National Technical Information Service, distributor.

Tuia, D., M. Volpi, et al., 2011. "A Survey of Active Learning Algorithms for Supervised Remote Sensing Image Classification." Selected Topics in Signal Processing, IEEE Journal of5(3): 606-617.

Ves, E. d., X. Benavent, et al., 2006. "Selecting the structuring element for morphological texture classification." Pattern Anal. Appl.9(1): 48-57.

Wang, X., 2009. Image Edge Detection Based on Adaptive Selection the Value of Structure Element of Mathematical Morphology. Electronic Computer Technology, 2009 International Conference on.

Wee C. and Paramesran, R. (2007). On The Computational Aspects of Zernike Moments. Image and Vision Computing, 25, pp967-980.

XIAO, X.M., Boles., S., Frolking, S., Li,C.S.,Babu, J.Y., Salas, W. and Moore, B., 2006, Mapping paddy rice agriculture in South and Southeast Asia using multi-temporal MODIS images. Remote Sensing of Environment, 100, 95-113.

Xiuming, J. and W. Jing., 2011. Research of remote sensing classification about land survey based on SVM. Artificial Intelligence, Management Science and Electronic Commerce (AIMSEC), 2011 2nd International Conference on.

Xiao-hong, Z.-y. Zhu, et al., 2009. Region of Interest Automatic Extraction for Color Image Based on Mathematical Morphology.
Y.H. Tseng., P.H. Hsu and I.H Chen., 1998. Asian conference on remote sensing "Automatic recognition of rice fields from multitemporal satellite images",

http://citeseerx.ist.psu.edu/viewdoc/summary?doi=10.1.1.48.64 52 